\title{
SIX1 induces lymphangiogenesis and metastasis via upregulation of VEGF-C in mouse models of breast cancer
}

\author{
Chu-An Wang, ${ }^{1}$ Paul Jedlicka, ${ }^{2}$ Aaron N. Patrick, ${ }^{3,4}$ Douglas S. Micalizzi, ${ }^{1,5}$ Kimberly C. Lemmer, ${ }^{1}$ \\ Erin Deitsch, ${ }^{3}$ Matias Casás-Selves, ${ }^{1}$ J. Chuck Harrell, ${ }^{6}$ and Heide L. Ford1,3,4,5,6 \\ 1Program in Molecular Biology, ${ }^{2}$ Department of Pathology, ${ }^{3}$ Department of Obstetrics and Gynecology, ${ }^{4}$ Department of Pharmacology, \\ ${ }^{5}$ Medical Scientist Training Program, and ${ }^{6}$ Program in Reproductive Sciences, University of Colorado Denver School of Medicine, Aurora, Colorado, USA.
}

\begin{abstract}
An association between lymph node metastasis and poor prognosis in breast cancer was observed decades ago. However, the mechanisms by which tumor cells infiltrate the lymphatic system are not completely understood. Recently, it has been proposed that the lymphatic system has an active role in metastatic dissemination and that tumor-secreted growth factors stimulate lymphangiogenesis. We therefore investigated whether SIX1, a homeodomain-containing transcription factor previously associated in breast cancer with lymph node positivity, was involved in lymphangiogenesis and lymphatic metastasis. In a model in which human breast cancer cells were injected into immune-compromised mice, we found that SIX1 expression promoted peritumoral and intratumoral lymphangiogenesis, lymphatic invasion, and distant metastasis of breast cancer cells. SIX1 induced transcription of the prolymphangiogenic factor VEGF-C, and this was required for lymphangiogenesis and lymphatic metastasis. Using a mouse mammary carcinoma model, we found that VEGF-C was not sufficient to mediate all the metastatic effects of SIX1, indicating that SIX1 acts through additional, VEGF-Cindependent pathways. Finally, we verified the clinical significance of this prometastatic SIX1/VEGF-C axis by demonstrating coexpression of SIX1 and VEGF-C in human breast cancer. These data define a critical role for SIX1 in lymphatic dissemination of breast cancer cells, providing a direct mechanistic explanation for how VEGF-C expression is upregulated in breast cancer, resulting in lymphangiogenesis and metastasis.
\end{abstract}

\section{Introduction}

The Six family member, Six1, was first identified as a mammalian homolog of the Drosophila sine oculis (so) gene and is highly conserved in numerous invertebrate and vertebrate species (1). The Six 1 gene encodes a homeodomain-containing transcription factor that is highly expressed in multiple tissues throughout embryogenesis, in which it plays an important role in the expansion of progenitor cell populations, in part through its ability to activate known regulators of the cell cycle, such as c-Myc $(2,3)$ and Gdnf (4). In most adult tissue, Six1 is not highly expressed; however, increased Six1 expression has been documented in multiple cancers. Importantly, reexpression of Six 1 in cancer can transcriptionally activate a set of protumorigenic genes, including cyclin D1, c-Myc, and Ezrin in rhabdomyosarcoma cells (5) as well as cyclin A1 (6) and type I transforming growth factor- $\beta$ receptor (7) in mammary epithelial and breast cancer cells, and its overexpression is transforming both in vitro and in vivo $(8,9)$. In addition, SIX1 overexpression correlates with poor prognosis in numerous tumor types, including breast cancer (10), suggesting that it plays an important role not only in tumorigenesis, but also in metastasis. Indeed, SIX1 is critical for metastasis in different models of cancer, including rhabdomyosarcoma (11), hepatocellular carcinoma (12), and breast cancer (10). Using an experimental metastasis model, we recently demonstrated that SIX1 plays a role in the later stages of metastasis through its ability to upregulate TGF- $\beta$ signaling (10). However, because SIX1 overexpression in MCF7 cells leads primarily to lymphatic metastasis in an ortho-

Conflict of interest: The authors have declared that no conflict of interest exists. Citation for this article: J Clin Invest. 2012;122(5):1895-1906. doi:10.1172/JCI59858. topic mouse model of mammary cancer, we hypothesized that it may additionally promote early stages of metastasis, such as the spread of tumor cells to the lymphatics.

The presence of tumor cells in regional lymph nodes (RLNs) is the main negative prognostic factor for breast cancers $(13,14)$. However, the mechanism by which tumor cells reach RLNs is still a subject of debate, as this may occur due to increased invasiveness of tumor cells, interactions between the tumor cells and the microenvironment, or merely by passive transport of the tumor cells via the preexisting lymphatic vessels. Nonetheless, growing evidence suggests that lymph node metastases may be facilitated by tumorstimulated lymphangiogenesis, the formation of newly developed lymphatic vessels within or surrounding the neoplastic lesion (15). Indeed, several animal studies have demonstrated that a range of lymphangiogenic growth factors, such as VEGF family members, FGF, or PDGF, are able to induce lymphangiogenesis or promote lymphatic metastasis (16-19). Importantly, clinical evidence supports the role of VEGF family members as the major lymphangiogenic regulators, because they are often associated with lymph node metastasis or lymphatic vessel density in breast carcinoma (20-22). VEGF-C and VEGF-D are specific lymphangiogenic factors, acting via activation of the VEGFR-3, which is expressed primarily on lymphatic endothelial cells. In lymphatic development, VEGF-C is required to trigger the sprouting of lymphatic vessels (23), while VEGF-D-deficient mice have no obvious lymphatic phenotype (24). These data suggest a predominant role for VEGF-C in stimulating lymphangiogenesis during development. Although lymphatic vessels are quiescent under physiological conditions in most adult tissues, production of lymphangiogenic factors by tumor cells or by inflammatory molecules can trigger 
A

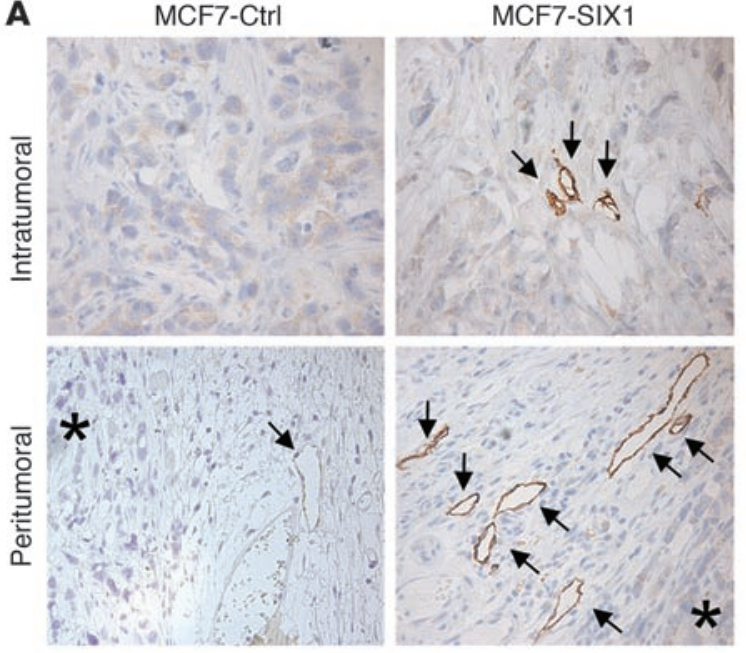

B

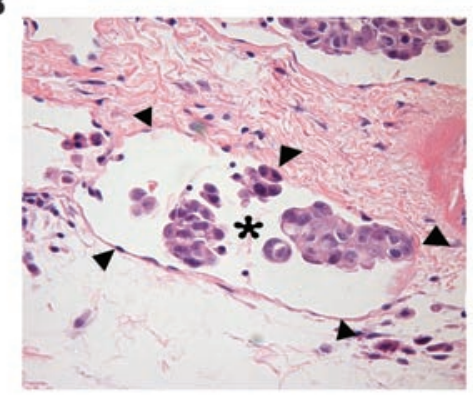

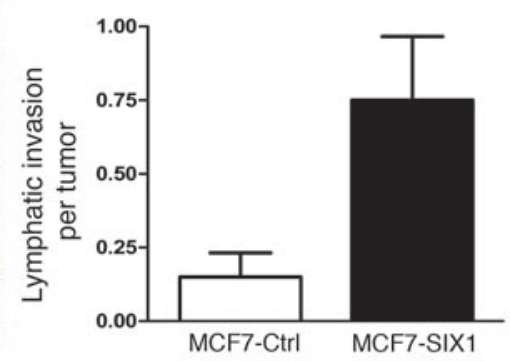

Figure 1

SIX1 overexpression leads to increased tumor-associated lymphangiogenesis and lymphatic invasion. (A) SIX1-expressing tumors have an increased number of lymphatic vessels, both within the neoplastic mass (intratumoral) and within the surrounding fibroinflammatory tissue (peritumoral), compared with that in control tumors of the same size. Positive immunostaining (brown coloration, DAB) for the lymphatic marker Lyve-1 was used to identify lymphatic vessels. Representative examples of intratumoral (original magnification, $\times 400$ ) and peritumoral (original magnification, $\times 200$ ) lymphatic vessels, as indicated by the arrows (asterisks represent tumors). Quantification of Lyve-1-positive vessels in a subset $(n=12)$ of the tumors. (B) Example of peritumoral lymphatic invasion by tumor cells (H\&E stain; asterisk represents tumor cells; arrowheads represent lymphatic vessels) (original magnification, $\times 400$ ). Quantification of the amount of peritumoral lymphatic invasion in histologic sections of MCF7$\mathrm{Ctrl}$ and MCF7-SIX1 tumors. All tumors were fixed when they reached a volume of $2 \mathrm{~cm}^{3} ; \mathrm{H} \& \mathrm{E}$-stained histologic sections were scored in a blinded manner by a pathologist. ${ }^{*} P<0.05$. lymphangiogenesis. Indeed, overexpression of VEGF-C strongly promotes the growth of tumor-associated lymphatic vessels in an MCF7 orthotopic model of mammary carcinoma $(25,26)$. Furthermore, in a second orthotopic model of breast cancer using MDA-MB-435 cells, overexpression of VEGF-C leads not only to a significant induction of intratumoral lymphangiogenesis, but also to RLN as well as distant lung metastases (27). Together, these data demonstrate that VEGF-C is an important regulator of lymphangiogenesis and lymphatic dissemination in breast cancer. However, little is known about the mechanism by which VEGF-C is regulated during tumorigenesis.

In this study, we demonstrate that SIX1 induces lymphangiogenesis and lymphatic/distant metastasis in an orthotopic mouse model of mammary carcinoma and that it does so by transcriptionally regulating a potent lymphangiogenic factor, VEGF-C. We fur- ing that SIX1 expression correlates with positive lymph node status in human breast cancer, led us to examine whether SIX1 could regulate lymphangiogenesis, thereby enhancing lymphatic metastasis. We thus examined MCF7-SIX1 and MCF7-control (MCF7-Ctrl) tumors for the presence of lymphatic vessels by staining tumor sections for Lyve-1 (hyaluronan receptor). Accordingly, MCF7-SIX1 tumors displayed an increased number of lymphatic vessels both within the tumors (intratumoral) and in the fibroinflammatory tissue adjacent to the tumor (peritumoral) (Figure 1A). Increased intratumoral lymphangiogenesis in MCF7-SIX1 tumors was also observed in more severely immunocompromised NOD/SCID mice (Supplemental Figure 2). Furthermore, MCF7-SIX1 tumors exhibited a 5-fold increase in lymphatic invasion at the tumor periphery compared with that in the MCF7-Ctrl tumors (Figure 1B). These data demonstrate that SIX1 is able to induce lymphangiogenesis and further sug- 

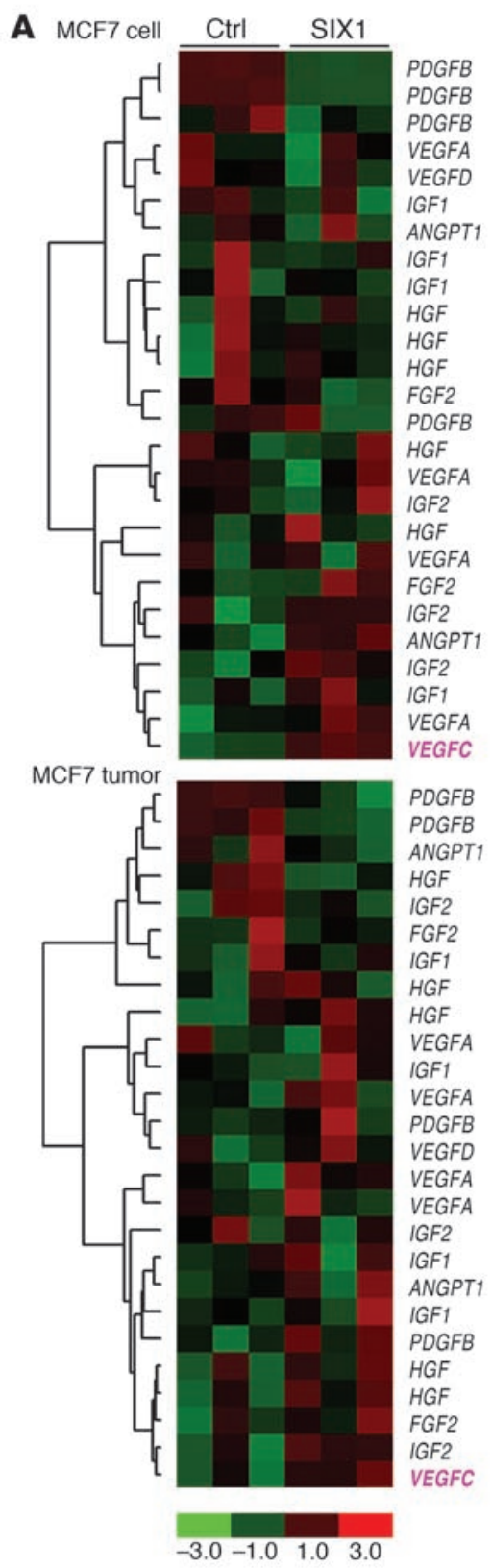
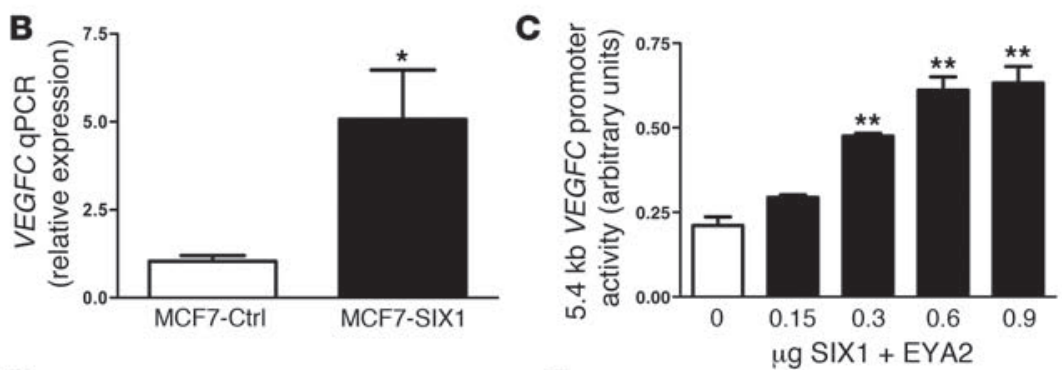

D

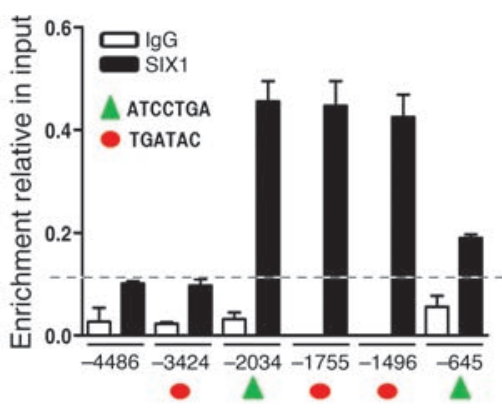

E

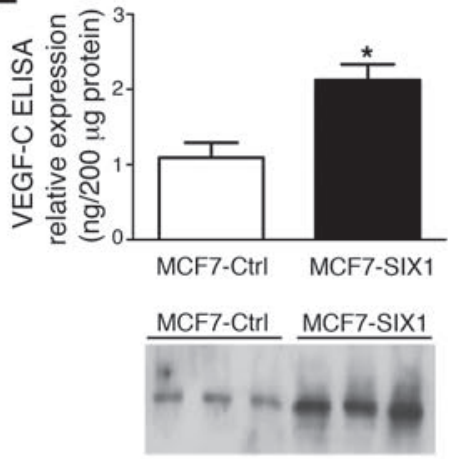

$\mathbf{F}$

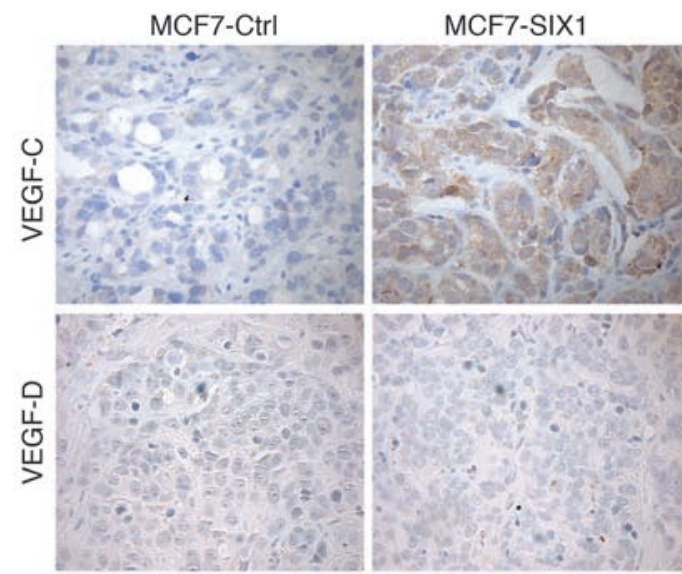

\section{Figure 2}

SIX1 transcriptionally activates VEGFC. (A) Microarray analysis demonstrates that SIX1 overexpression leads to increased VEGFC mRNA in both MCF7-SIX1 cells and MCF7-SIX1 tumors. (B) Quantitation of VEGFC gene expression in MCF7-Ctrl and MCF7-SIX1 using real-time PCR. (C) SIX1 induces VEGFC promoter activity. VEGFC promoter-luciferase reporter constructs were transiently transfected into MCF7 cells, along with increasing amounts of SIX1 and a constant amount of the SIX1 cofactor, EYA2. Luciferase activity was analyzed after 48 hours. (D) ChIP was performed to detect SIX1 presence on the VEGFC promoter in MCF7 cells transfected with SIX1 and EYA2. Protein-DNA complexes were precipitated with a SIX1-specific antibody as well as a control rabbit IgG antibody, after which real-time PCR was performed with primers that flank 5 predicted SIX1 binding sites within the VEGFC promoter (red circles denote the TGATAC binding sites; green triangles denote the ATCCTGA binding sites) as well as 1 upstream region with no predicted SIX1 binding site as a negative control. Dashed line indicates the background non-specific binding of SIX1 and $x$ axis units are base pairs upstream of transcription start site. (E) Functional VEGF-C secreted by MCF7-Ctrl and MCF7-SIX1 cells was measured by ELISA and Western blot analysis ( 3 clonal isolates of MCF7-Ctrl cells [lanes 1-3] and 3 clonal isolates of MCF7-SIX1 cells [lanes 4-6]) in conditioned medium after serum starvation for 48 hours. (F) MCF7SIX1 tumors express higher levels of VEGF-C in vivo compared with the MCF7-Ctrl tumors. Immunostaining was used to detect VEGF-C and VEGF-D. Original magnification, $\times 400 .{ }^{*} P<0.05 ;{ }^{* \star} P<0.01$. 

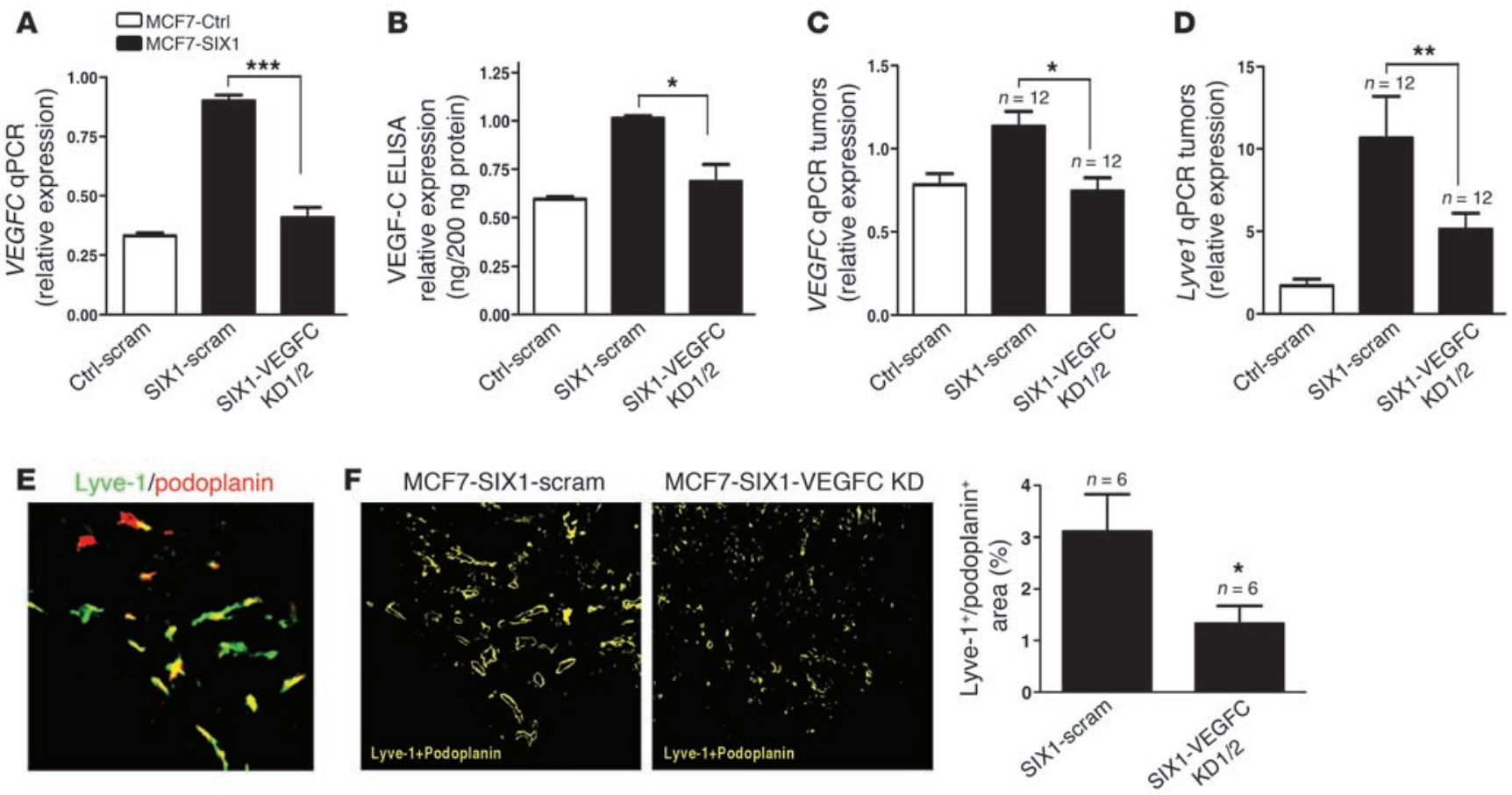

Figure 3

VEGF-C mediates SIX1-induced lymphangiogenesis. (A) VEGFC expression in MCF7-Ctrl, MCF7-SIX1, and MCF7-SIX1-VEGFC KD cells was quantified by real-time PCR. Two shRNAs (VEGFC KD1 and VEGFC KD2; data regarding VEGFC KD1 and VEGFC KD2 are labeled VEGFC $\mathrm{KD} 1 / 2$ in the figure) were used to knockdown VEGF-C in MCF7-SIX1 cells, back to the level observed in MCF7-Ctrl cells. A scramble shRNA was introduced into MCF7-Ctrl (Ctrl-scram) and MCF7-SIX1 (SIX1-scram) cells to serve as a control. (B) Secreted VEGF-C in cultured medium from MCF7-Ctrl, MCF7-SIX1, and MCF7-SIX1-VEGFC KD cells was measured by ELISA. (C) VEGFC expression in MCF7-Ctrl, MCF7-SIX1, MCF7SIX1-VEGFC KD tumors was quantified by real-time PCR. (D) Lyve1 expression in MCF7-Ctrl, MCF7-SIX1, MCF7-SIX1-VEGFC KD tumors was quantified by real-time PCR. (E) Representative image shows immunofluorescence for Lyve-1 (green) and podoplanin (red) in MCF7-SIX1 tumors. Original magnification, $\times 200$. (F) SlideBook software was used to transfer the Lyve-1/podoplanin double-stained regions (as shown in E) in MCF7-SIX1 and MCF7-SIX1-VEGFC KD tumors to yellow signals as shown. Original magnification, $\times 200$. Quantification of lymphatic vessels in MCF7-SIX1 and MCF7-SIX1-VEGFC KD tumors. ${ }^{*} P<0.05 ;{ }^{* \star} P<0.01 ;{ }^{* * *} P<0.001$.

gest that SIX1-induced lymphangiogenesis may contribute to the ability of SIX1 to promote lymphatic metastasis.

SIX1 is a transcriptional regulator of VEGF-C. To determine the mechanism by which SIX1 promotes lymphangiogenesis, we examined previously obtained gene expression data sets from MCF7-SIX1 or MCF7-Ctrl cells (10), as well as from MCF7-SIX1 and MCF7Ctrl tumors, for tumor-related lymphangiogenic factors. Among the known lymphangiogenic factors, VEGFC was consistently upregulated in both MCF7-SIX1 cells and MCF7-SIX1 tumors compared with the MCF7-Ctrl cells and MCF7-Ctrl tumors (Figure 2A). The SIX1-induced upregulation of VEGFC was confirmed in independent RNA samples using quantitative real-time PCR (Figure 2B); while the mRNA expression of 2 other reported major lymphangiogenic factors, VEGFA and VEGFD, was not significantly changed (Supplemental Figure 3). Because SIX1 is a transcription factor, we examined whether it upregulates $V E G F C$ via activating its promoter. Analysis of the activity of a VEGFC promoter-luciferase construct in response to increasing doses of SIX1 and its required cofactor EYA2 $(2,28)$ indicated that SIX1 can induce the VEGFC promoter in a dose-dependent manner (Figure 2C). Further inspection of the VEGFC promoter revealed 5 predicted SIX1 binding sites $(2,29)$, suggesting that SIX1 directly activates VEGFC. To determine whether SIX1 binds to the VEGFC promoter in the context of chromatin, we performed ChIP assays. SIX1 and its cofactor EYA were transfected into MCF7 cells and DNA-protein complexes were immunoprecipitated using a SIX1-specific or control IgG antibody. Primers (see Supplemental Methods) spanning the 5 candidate SIX1 binding regions were then used to perform real-time PCR, along with PCR primers (see Supplemental Methods) that span a region upstream of the VEGFC promoter ( -4486 to $-4384 \mathrm{bp}$ ) with no predicted SIX1 binding site. These studies demonstrated that SIX1 binds primarily to 3 adjacent predicted binding sites on the VEGFC promoter (Figure 2D). However, due to the close proximity of these 3 sites to each other, and the concern that we were unable to distinguish whether binding occurred preferentially to one site versus the others, we further performed electromobility gel shift assays with wild-type sequences as well as with mutated competitors to identify whether SIX1 preferentially and specifically binds to 1 of the 3 sites identified in our ChIP assay. As shown in Supplemental Figure 4, the central of the 3 sites (site at -1755 bp, Figure 2D), which contains the core SIX1 binding sequence (TGATAC) and associated flanking regions, was specifically bound by SIX1. Taken together, these data demonstrate that SIX1 binds specific DNA sequences in the VEGFC promoter, both in vitro and in vivo, and that it directly activates VEGFC transcription. Because SIX1 transcriptionally regulates VEGFC, we further examined whether the increase in VEGFC mRNA lead to an increase in VEGF-C protein. Indeed, SIX1 overexpression in MCF7 


\section{Table 1}

Effect of SIX1 and VEGF-C on lymphatic spread and metastasis

\begin{tabular}{lccc} 
& MCF7-Ctrl-scramble KD & MCF7-SIX1-scramble KD & MCF7-SIX1-VEGFC KD1/2 \\
Mice with lung metastasis & $0 \%(0 / 13)$ & $18 \%(4 / 22)$ & $0 \%(0 / 18)$ \\
Mice with multiple positive lymph nodes $(\geq 2)$ & $0 \%(0 / 13)$ & $59 \%(13 / 22)^{\mathrm{A}}$ & $11 \%(2 / 18)^{\mathrm{A}}$ \\
\hline Fisher's exact test $(2$ sided) indicates a significant decrease in the number of mice with multiple involved lymph nodes, if they were bearing MCF7-SIX1- \\
VEGFC KD tumors as compared with MCF7-SIX1 tumors. In addition, distant metastasis was completely abrogated in mice bearing MCF7-SIX1-VEGFC \\
KD tumors. ${ }^{A} P=0.0028$.
\end{tabular}

cells resulted in increased secretion of VEGF-C protein into the medium, as detected using both ELISA and Western blot analysis (Figure 2E). In addition, increased VEGF-C protein was observed in the MCF7-SIX1 tumors compared with that in the MCF7-Ctrl tumors, while another related-lymphangiogenic factor, VEGF-D, was not detectable (Figure 2F).

VEGF-C mediates SIX1-induced lymphangiogenesis in vivo. Mice injected orthotopically with MCF7-SIX1 cells display a similar lymphatic metastasis phenotype to those injected with MCF7 cells that overexpress VEGF-C $(25,26)$, suggesting that SIX1 is dependent on VEGF-C to induce metastasis. To determine whether SIX1 depends on VEGF-C to promote lymphatic metastasis, we used 2 independent shRNAs to knockdown VEGF-C in the MCF7-SIX1 cells. In addition, we introduced a scrambled shRNA into both MCF7-Ctrl and MCF7-SIX1 cells to serve as an off-target control. Importantly, the VEGF-C knockdown restored VEGF-C levels to those observed in MCF7-Ctrl cells (Figure 3, A and B). MCF7-Ctrl scrambled knockdown (MCF7-Ctrl-scramble KD), MCF7-SIX1 scrambled KD (MCF7-SIX1-scramble KD), and MCF7-SIX1-VEGFC KD cells were labeled with Zsgreen to enhance our ability to visualize lymph node metastases. The cells were injected into the fourth mammary fat pad of nude mice, after which tumors were allowed to grow to a uniform size of $2 \mathrm{~cm}^{3}$. Primary tumors were examined for $V E G F C$ levels by real-time PCR, confirming that VEGF-C knockdown was maintained in the tumors (Figure $3 \mathrm{C}$ ). No difference in primary tumor growth was observed between MCF7-SIX1-scramble KD and MCF7-SIX1-VEGFC KD tumors (Supplemental Figure 5A). However, knockdown of VEGF-C in MCF7-SIX1 tumors significantly reversed lymphangiogenesis, as measured by Lyve1 mRNA expression, nearly back to the levels observed in MCF7-Ctrl tumors (Figure 3D), demonstrating a dependence of SIX1 on VEGF-C to stimulate lymphatic vessel formation. Because Lyve- 1 can in some instances be found on macrophage-like cells (30), we further confirmed that SIX1 depends on VEGF-C to increase the number of lymphatic vessels within a tumor by double staining the MCF7SIX1-scramble and MCF7-SIX1-VEGFC KD tumors with both Lyve-1 and an additional lymphatic vessel marker, podoplanin (a mucin-type transmembrane glycoprotein expressed by lymphatic endothelial cells). A representative double immunofluorescence image is shown in Figure 3E. Areas that stained positively for both Lyve-1 and podoplanin were identified as lymphatic vessels in MCF7-SIX1-scramble KD and MCF7-SIX1-VEGFC KD tumors and were quantified using SlideBook software, conclusively demonstrating a decrease in lymphatic vessel formation (lymphangiogenesis) in MCF7-SIX1-VEGFC KD tumors compared with that in the MCF7-SIX1-scramble KD control tumors (Figure 3F).

SIX1 is dependent on VEGF-C to induce metastasis. Because increased VEGF-C and lymphangiogenesis are both associated with increased metastasis (15), we examined whether SIX1 is dependent on VEGF-C to mediate metastasis in our MCF7 orthotopic model of mammary cancer. We observed a decrease in distant metastases to the lungs, with $0 \%$ observable distant metastases found in animals bearing MCF7-SIX1-VEGFC KD tumors ( 0 out of 18 ), whereas $18 \%$ (4 out of 22) of animals bearing MCF7-SIX1-scramble KD tumors had observable distant metastases (identified by ZsGreen signal in the lung and selectively confirmed by histology; Supplemental Figure 5B) (Table 1). Clinically, the number of positive lymph nodes within a patient correlates with distant metastasis; therefore, we further analyzed whether the number of positive lymph nodes per mouse was altered with VEGF-C KD. While SIX1 overexpression in MCF7 tumors leads to an increase in the number of positive lymph nodes per mouse when compared with MCF7-Ctrl tumors, this increase is significantly diminished when VEGF-C is knocked down in the context of SIX1 overexpression ( $P=0.0028,2$-tailed, Fisher's exact test) (Table 1$)$. These data strongly support the hypothesis that SIX1-induced VEGF-C expression is necessary for lymphatic dissemination, ultimately resulting in distant metastasis.

Vegf-c expression is required for lymphatic and distant metastasis in the $66 c 14$ mouse mammary carcinoma model. Although levels of exogenous SIX1 protein in the MCF7 cells do not exceed endogenous levels of SIX1 seen in other human breast cancer cell lines, suggesting that SIX1 expression in our MCF7 model is within the physiologic range observed in cancer (Supplemental Figure 6), we nonetheless sought a complementary model in which we knocked down Six1, rather than overexpressed it, to ensure that the phenotypes observed with SIX1 overexpression corroborate those seen in an endogenous context. Thus, we turned to the $66 \mathrm{cl} 4$ mouse mammary carcinoma model of metastasis. $66 \mathrm{cl} 4$ cells were derived as a subline from a spontaneous mammary carcinoma in Balb/c mice, and these cells metastasize to the lungs primarily through the lymphatics (as opposed to through the vasculature) (31). Although it is known that $66 \mathrm{cl} 4$ cells metastasize mainly through the lymphatic system, the role of Vegf-c in mediating metastasis of $66 \mathrm{cl} 4$ cells has not previously been examined. To determine whether Vegf-c is required for $66 \mathrm{cl} 4$ cells to metastasize from the orthotopic site, we stably knocked down Vegf-c using 2 different shRNA containing constructs (Supplemental Figure 7, A and B). 66cl4 scrambled KD (66cl4-scramble $\mathrm{KD})$ and $66 \mathrm{cl} 4-\mathrm{Vegf-c} \mathrm{KD}$ cells were then luciferase tagged to allow monitoring of metastasis in vivo over time. Forty days after injection of the cells into the fourth mammary fat pad of Balb/c mice, we observed a dramatic decrease in lung metastasis when Vegf-c was knocked down (6\%, 1 out of 15 mice) compared with that in the $66 \mathrm{cl} 4$-scramble group, for which $100 \%$ of the mice had metastases (10 out of 10 mice), demonstrating that Vegf-c mediates the metastasis observed in this model (Supplemental Figure 7C). However, it should be noted that we also observed differences in the growth rate of the primary tumors between 
A

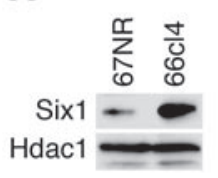

B
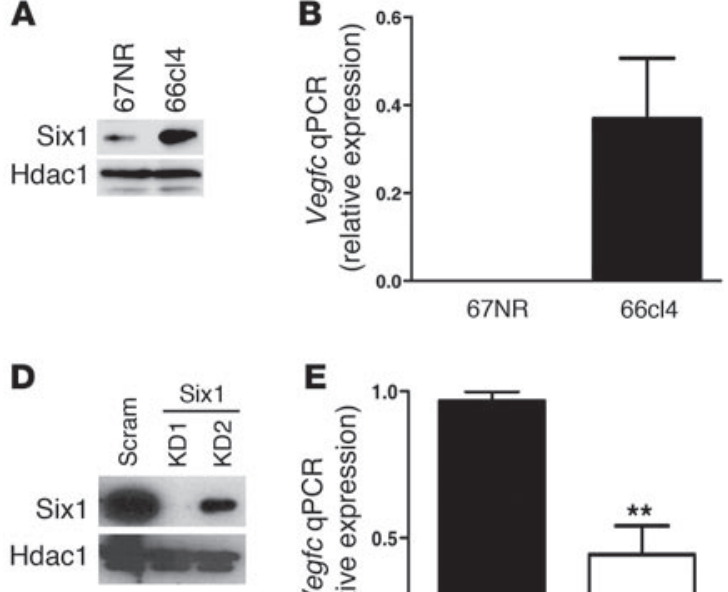

E

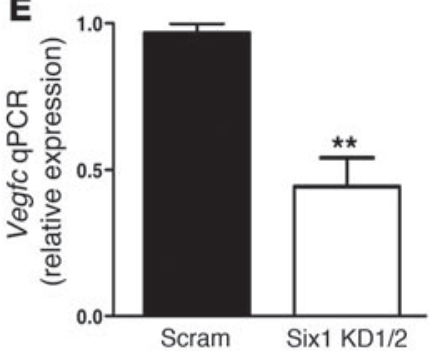

C

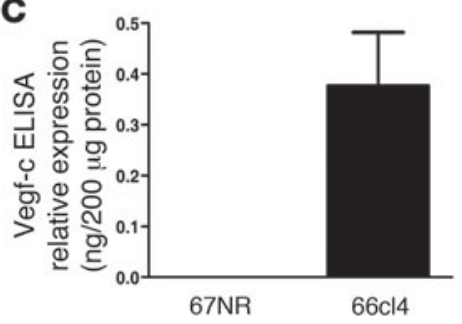

$\mathbf{F}$

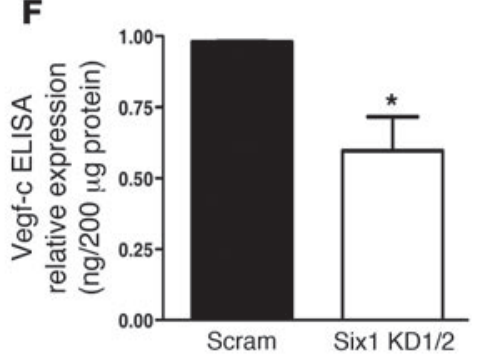

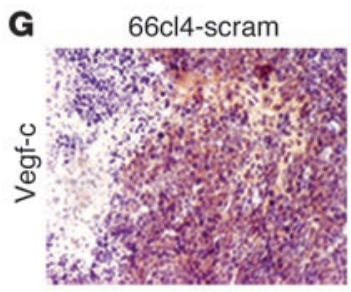

66cl4-Six1 KD
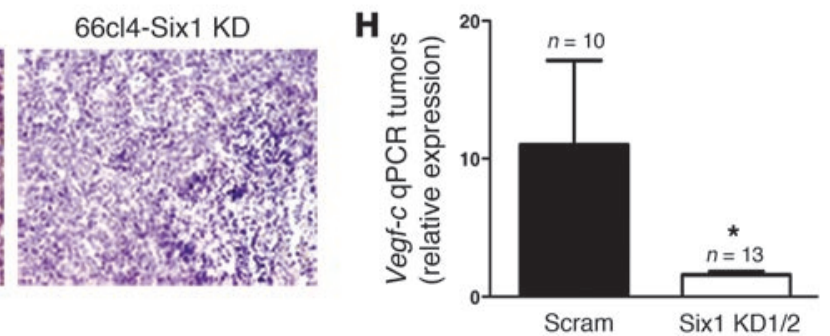

I
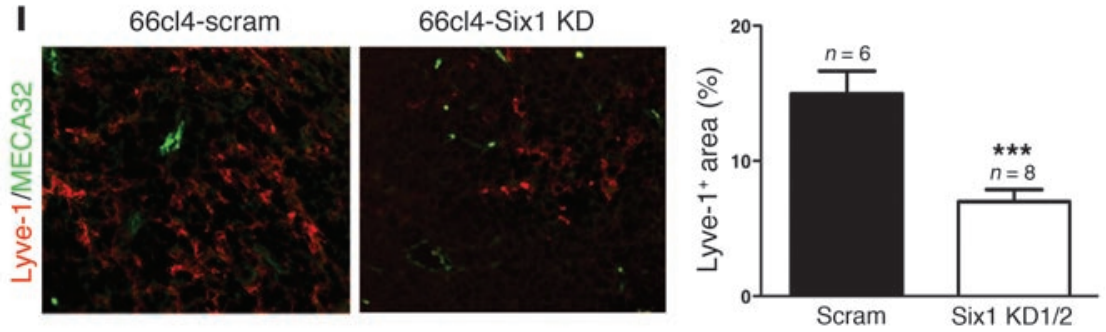

Figure 4

Knockdown of Six 1 in $66 \mathrm{cl} 4$ mammary carcinoma cells leads to a reduction in Vegf-c and lymphangiogenesis. (A) Western blot analysis demonstrates increased Six1 expression in the metastatic 66cl4 cell line as compared with that in the syngeneic, nonmetastatic 67NR cell line. (B) Real-time PCR demonstrates that Vegfc mRNA is increased in $66 \mathrm{cl} 4$ cells as compared with that in 67NR cells. (C) $66 \mathrm{cl} 4$ cells contain high levels of Vegf-c in their conditioned media, as assessed by ELISA. (D) Expression of Six1 in $66 \mathrm{cl} 4$ cells expressing either a control (scram) or Six1 knockdown (KD1/KD2) construct. Two shRNAs were used to knockdown Six1 and are individually shown in the panel while shown as Six1 KD1 and Six1 KD2 in the following panels. (E) Vegfc mRNA is decreased in 66cl4-Six1 KD cells relative to that in 66cl4-scramble cells, as measured by real-time PCR. (F) Vegf-c protein is decreased in the conditioned media from $66 \mathrm{cl} 4-S i x 1 \mathrm{KD}$ cells relative to that in $66 \mathrm{cl} 4$-scramble cells, as measured by ELISA. (G) 66cl4-Six1 KD tumors express less Vegf-c, as detected by immunostaining, than 66cl4scramble (66cl4 scramble) control tumors. Representative images are shown. Original magnification, $\times 200$. (H) Real-time PCR demonstrates that $66 \mathrm{cl} 4-$ Six1 KD tumors contain less Vegfc mRNA than the 66cl4-scramble control tumors. (I) 66cl4-scramble and Six1 KD tumors were stained with antibodies against Lyve-1 (red) and MECA32 (green), and representative images are shown. Original magnification, $\times 400$. SlideBook software was used to quantify Lyve-1-positive, MECA32-negative regions in 66cl4-scramble and 66cl4-Six1 KD tumors. ${ }^{*} P<0.05 ;{ }^{* *} P<0.01 ;{ }^{* \star *} P<0.001$.

the 66cl4-scramble KD and 66cl4-Vegf-c KD cells (Supplemental Figure 7D), suggesting that tumor-associated Vegf-c regulates not only lymphatic/distant metastasis in this model but that it also regulates primary tumor growth.
Six1 is required for the induction of Veef-c and lymphangiogenesis in vivo. The $66 \mathrm{cl} 4$ mammary carcinoma cell line is an ideal model by which to examine whether tumor cells remain dependent on Six1 to promote lymphatic and distant metastasis. First, Six 1 is highly expressed in this cell line, in contrast to its expression in the syngeneic but nonmetastatic $67 \mathrm{NR}$ cell line (Figure 4A). Importantly, the $66 \mathrm{cl} 4$ cells also expressed high levels of Vegfc as opposed to other Vegf family members (Figure 4, B and C, and Supplemental Figure 8A), and, as we demonstrated above, they were dependent on Vegf-c to mediate metastasis. Thus, to test whether Six1 regulates Vegfc in 66cl4 mouse mammary carcinoma cells and to further determine whether Six 1 is required for metastasis in this system, we stably knocked down Six1 using 2 different shRNA constructs (Six1KD1 and Six1 KD2) (Figure 4D. In support of our hypothesis, we observed decreased Vegfc expression in Six 1 knockdown cells (Figure 4, E and F) and no significant change of Vegfa or Vegfd expression (Supplemental Figure 8B). To further strengthen the argument that Six 1 transcriptionally regulates $V e g f c$ in an endogenous setting, we performed a ChIP assay to determine whether endogenous Six 1 binds to the mouse Vegfc promoter. Supplemental Figure 9 demonstrates that endogenous Six 1 bound to a region within the mouse $\operatorname{Veg} f c$ promoter that contains 2 predicted Six 1 binding sites, demonstrating that Six 1 is bound to the Vegfc promoter in the mouse in addition to the human and that it thus likely regulates transcription of $V e g f c$ in this endogenous context.

Control and Six1-knockdown cells were subsequently luciferase-tagged and injected into the fourth mammary fat pat of Balb/c mice. Forty days after injection, animals were sacrificed and tumors were analyzed. As expected, decreased Vegf-c levels were observed in 66cl4-Six1 $\mathrm{KD}$ tumors compared with those in the scramble control tumors (Figure 4, $\mathrm{G}$ and $\mathrm{H}$ ). To determine whether decreased Vegf-c expression in Six1 KD tumors correlates with decreased lymphangiogenesis, tumors were double stained with Lyve-1 and MECA32 (mouse endothelial antigen-32, a marker of blood vessels), and intratumoral lymphangiogenesis was quanti- 
A

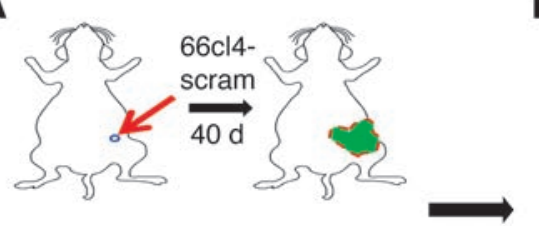

66cl4- Primary tumor

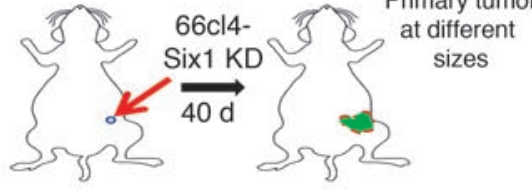

B

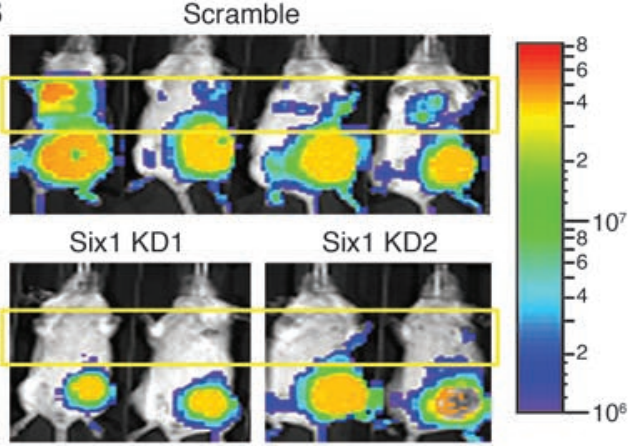

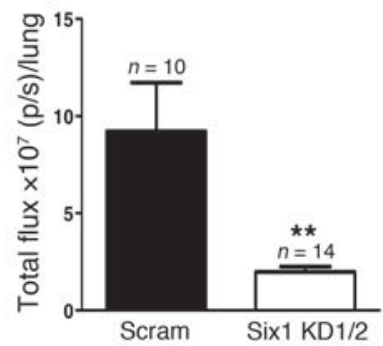

C

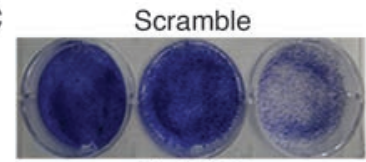

Six1 KD1

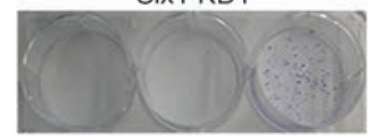

Scramble

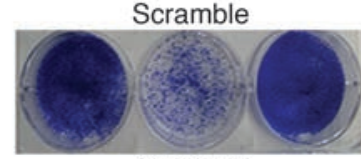

Six1 KD2

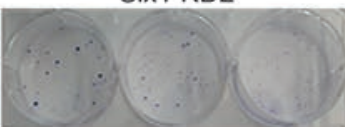

D
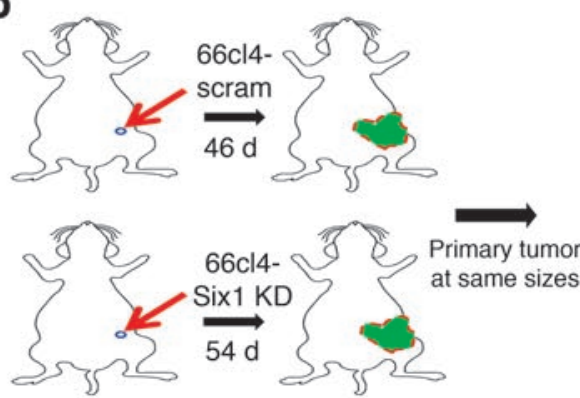

F

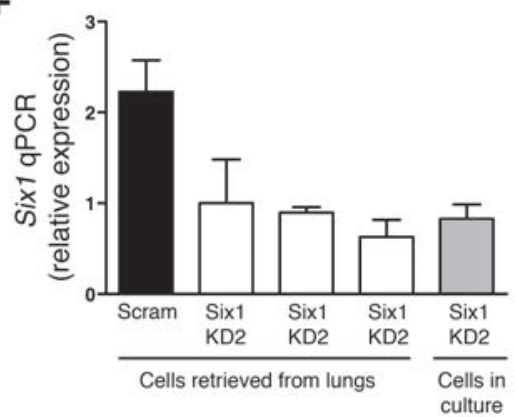

E

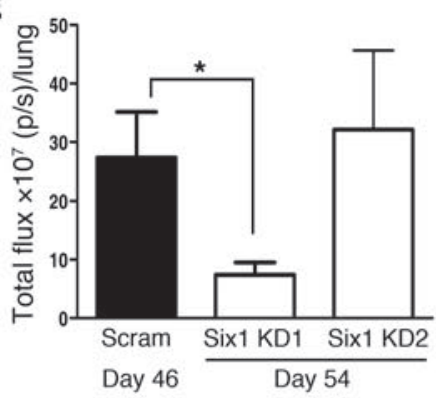

Figure 5

Six1 knockdown decreases distant metastasis, and the Six family member, Six2, likely compensates for loss of Six1. (A) Diagrammatic representation of the metastasis experiment performed in $\mathbf{A}-\mathbf{C}$, in which tumors were allowed to grow for the same amount of time before sacrifice. 66cl4-scramble or 66cl4-Six1 KD1/2 cells were injected into the fourth mammary fat pad of Balb/c mice, and metastases were measured weekly. (B) Representative bioluminescent imaging of Balb/c mice 40 days after injection of $66 \mathrm{cl} 4$-scramble or $66 \mathrm{cl} 4-\mathrm{Six} 1 \mathrm{KD} 1 / 2$ cells into the fourth mammary fat pad. Quantitation of distant luminescent signal, likely in lungs (yellow boxed region), in 66cl4-scramble and Six1 KD groups. p/s, photons per second. (C) Clonogenic assays were used to detect metastases in lungs of animals bearing 66cl4-scramble and 66cl4-Six1 KD tumors. Representative images from 66cl4-scramble and 66cl4-Six1 KD are shown. (D) Diagrammatic representation of the metastasis experiment performed in D-G, in which tumors were allowed to grow to the same size before sacrifice. 66cl4-scramble or 66cl4-Six1 KD1/2 cells were injected into the fourth mammary fat pad of Balb/c mice, and metastases were measured when tumor sizes were comparable. (E) Quantitation of bioluminescent imaging (photons per second) emanating from the region of lungs in Balb/c mice injected with 66cl4-scramble or 66cl4-Six1 KD1 and KD2 when tumors reached comparable sizes (day 46 for 66cl4-scramble and day 54 for 66cl4-Six1 KD1 and KD2). (F) Six1 expression was measured by real-time PCR in parental Six1 KD2 cells (in culture), Six1 KD2 cells retrieved from lungs of 3 individual mice, and 66cl4-scramble control cells retrieved from lung of 1 animal. The numbers over the bars represent Six2 expression fold change over the value of Scram. (G) Six2 expression was measured by real-time PCR in the above-mentioned cells. ${ }^{*} P<0.05 ;{ }^{*} P<0.01$.

fied by measuring Lyve-1-positive and MECA32-negative staining. Indeed, knockdown of Six 1 in 66 cl 4 cells resulted in decreased lymphangiogenesis in vivo (Figure 4I). To exclude the possibility that decreased Vegf-c in response to Six $1 \mathrm{KD}$ also plays a role in tumor-associated angiogenesis (32), 66cl4-scramble and Six1 KD tumors were stained with MECA32 and CD31 (PECAM-1, a marker strongly expressed by blood vessels). Quantification of the number of blood vessels demonstrated no significant differ- ence in angiogenesis between 66cl4-scramble and Six1 KD tumors (Supplemental Figure 10).

Vegf-c is sufficient to partially restore lymphangiogenesis and lymphatic metastasis in the absence of Six 1 but is not sufficient to restore distant metastasis. Examination of metastases in mice bearing 66cl4-scramble and 66cl4-Six1 KD tumors demonstrated a significant decrease in lung metastasis 40 days after orthotopic injection of the cells (Figure 5, A and B). To confirm that the luminescence seen with IVIS 
A

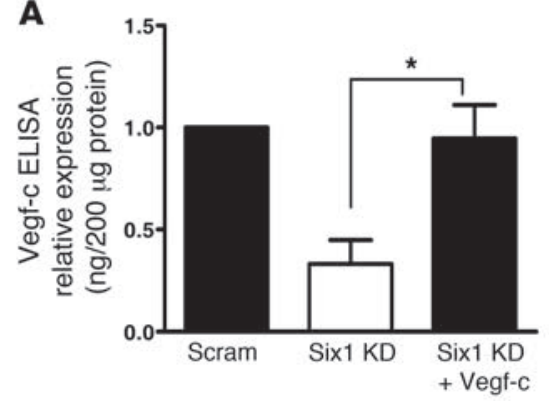

D

Scram

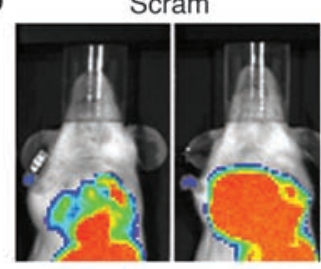

B

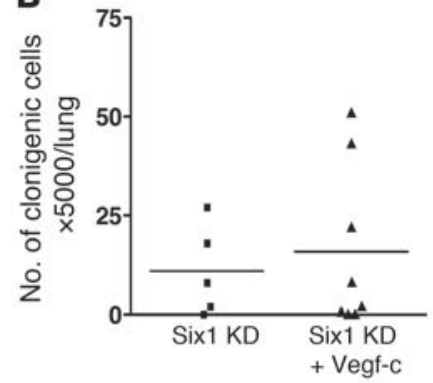

Six1 KD

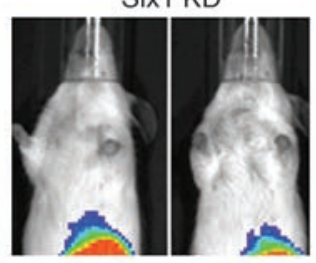

Six1 KD + Vegf-c

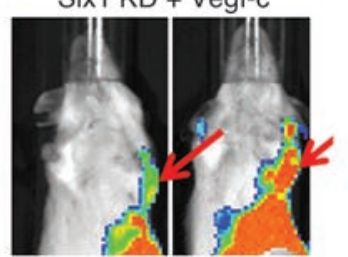

C
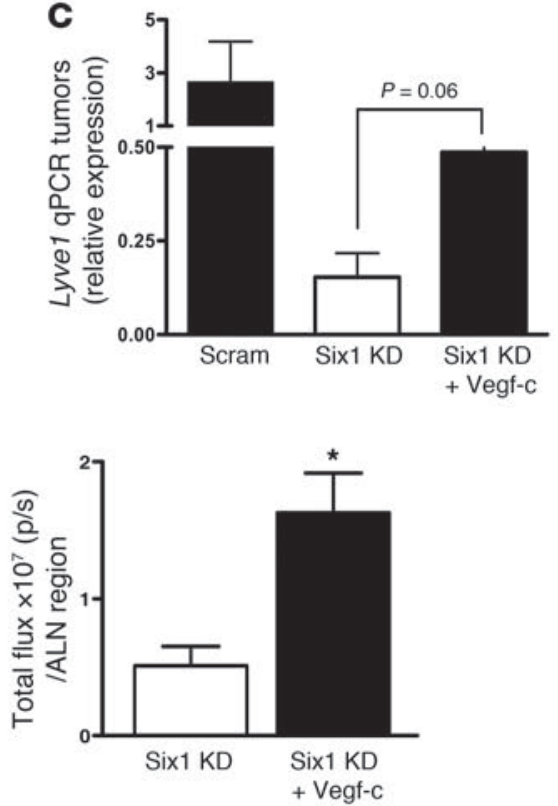

Figure 6

Six1 induces lymphangiogenesis and lymphatic metastasis, but not distant metastasis, in a Vegf-c-dependent manner. (A) Vegf-c ELISA performed on conditioned media from 66cl4-scramble, 66cl4-Six1 KD, and 66cl4-Six1 KD plus Vegf-c rescue (Six1 KD+Vegf-c) cells. (B) Clonogenic assays were used to detect metastasis in the lungs of animals bearing 66cl4-Six1 KD and 66cl4-Six1 KD plus Vegf-c tumors. Symbols represent individual mice, and the horizontal bars represent the mean. (C) Lyve1 mRNA expression in 66cl4-scramble, 66cl4-Six1 $\mathrm{KD}$, and 66cl4-Six1 KD plus Vegf-c tumors was determined using real-time PCR. (D) Representative bioluminescent imaging of Balb/c mice bearing 66cl4-scramble, 66cl4-Six1 KD, or Six1 KD plus Vegf-c tumors. Red arrows indicate positive luciferase signal around axillary lymph node regions (ALN). Quantitation of luciferase signal (photons per second) around the axillary lymph node regions in Six1 KD and Six1 KD plus Vegf-c groups. $\mathrm{p} / \mathrm{s}$, photons per second. ${ }^{*} P<0.05$.

imaging represented lung metastases, animals were sacrificed on day 40, and their lungs were dissected out. Lungs were then minced and collagenase digested, and the isolated cells were plated in the presence of 6-thioguanine, since $66 \mathrm{cl} 4$ cells are resistant to this drug (33). Ten days after 6-thioguanine selection, colonies were stained with crystal violet to determine the number of $66 \mathrm{cl} 4$ metastatic cells that were present in the lungs. Strikingly, we observed a dramatic difference in the number of 6-thioguanine-resistant cells isolated from 66cl4-scramble and 66cl4-Six1 KD lungs (Figure $5 \mathrm{C}$ ). Together, these data demonstrate that Six 1 plays a critical role in regulating Vegf-c levels and lymphangiogenesis and, most importantly, in regulating distant metastasis.

Interestingly, loss of Six1, similar to loss of Vegf-c, also resulted in a decrease in primary tumor growth (Supplemental Figure 11). This decrease in primary tumor growth rate due to Six1 KD could in part be due to the reduction of Vegf-c levels in the cells; however, as Six 1 is known to regulate several proliferative pathways $(3,6)$, the effects on proliferation are likely multifactorial. Clinically, larger tumor size is correlated with metastatic risk. To elucidate whether the decreased lung metastases observed in $66 \mathrm{cl} 4-\mathrm{Six} 1 \mathrm{KD}$ are due to a direct effect on metastasis and not due merely to alteration in tumor burden, we performed another set of experiments in which we injected 66cl4-scramble and 66cl4-Six1 $\mathrm{KD} 1 / 2$ cells orthotopically into Balb/c mice and then compared the metastatic burden when tumors reached the same size (Figure 5D and Supplemental Figure 12A). In this experiment, only the group in which Six1 was most efficiently knocked down (Six1 KD1) showed a significant decrease in lung metastasis compared with the scramble KD control (Figure 5E and Supplemental Figure 12B). To determine whether Six 1 knockdown was maintained in the other Six1 KD group (Six1 KD2) or whether the loss of the Six1 $\mathrm{KD}$ may explain the failure to diminish metastasis in this clone, we retrieved $66 \mathrm{cl} 4$ cells from the lungs of mice orthotopically injected with 66cl4-Six1 KD2 cells and performed real-time PCR for Six1 expression as well as for the expression of other Six family members. Surprisingly, although Six1 knockdown was maintained in retrieved cells (66cl4-Six1 KD2 cells), the expression level of Six2, a highly related Six family member, was increased in the metastatic lesions (Figure 5, F and G). In contrast, no alterations in other Six family members were observed (data not shown). Our findings strongly suggest that Six1 directly influences metastasis when tumors are grown to the same size and further suggest that other Six family members, such as Six 2 , may compensate for loss of Six 1 in mediating metastasis. Indeed, in the rare metastases observed in our Six1 KD1 clone, we also saw an increase in Six2 levels (Supplemental Figure 13).

Finally, to determine whether Vegf-c is sufficient to induce lymphatic and distant metastasis downstream of Six1, we restored Vegf-c expression (Vegf-c rescue) to 66cl4-Six1 KD1 cells at a level comparable to that found in parental 66cl4-scramble cells (Figure 6A). 66cl4-scramble cells plus pcDNA, 66cl4-Six1 KD cells plus pcDNA, and 66cl4-Six1 KD cells plus Vegf-c were injected into the fourth mammary gland of Balb/c mice, and in vivo metastases were examined. Forty days after injection, neither the 66cl4-Six1 KD plus pcDNA nor the Six1 KD plus Vegf-c tumor-bearing mice developed obvious metastases in the lungs, as measured with IVIS 
A
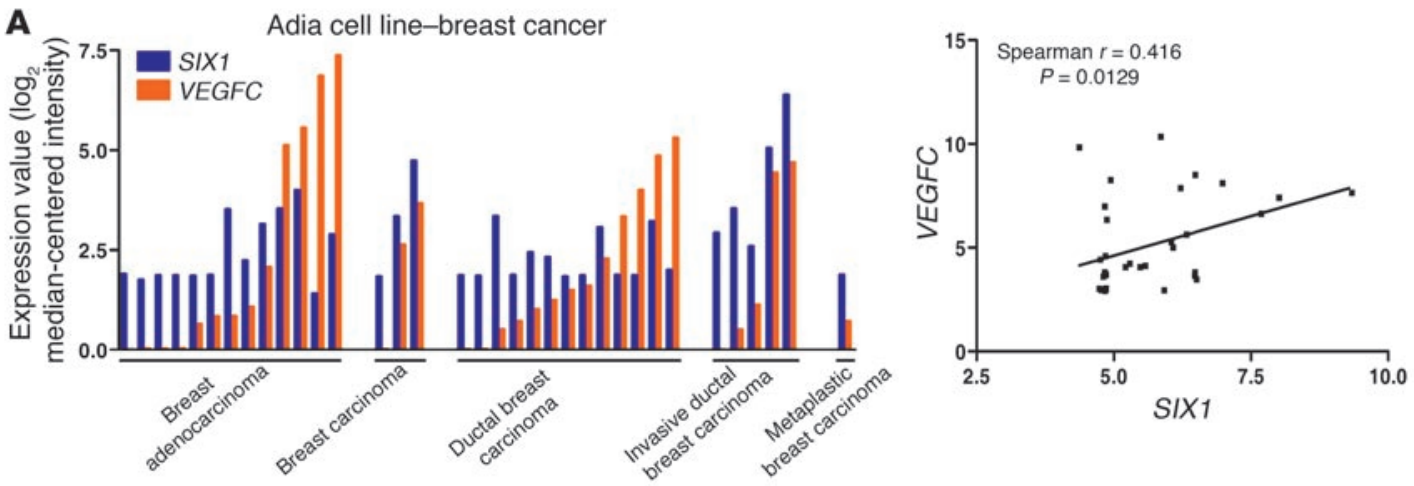

B

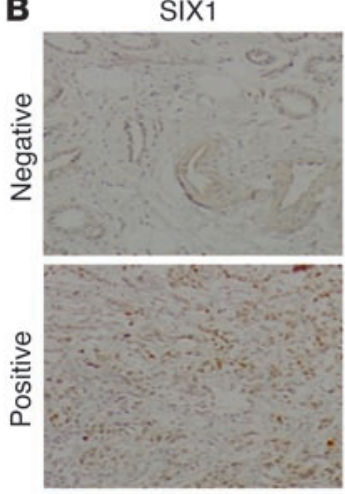

VEGF-C

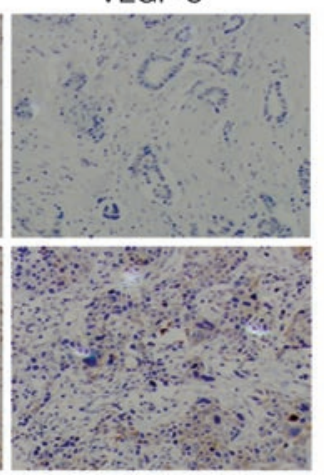

C

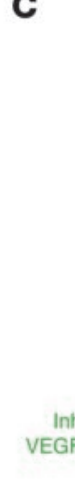

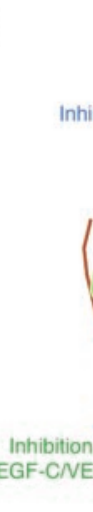

\section{Figure 7}

SIX1 correlates with VEGF-C expression in mammary carcinoma cell lines and in human breast cancers. (A) SIX1 and VEGFC expression values were retrieved from an Oncomine microarray data set (as indicated in the figure) and were plotted according to different types of cell lines or by expression value. $X$ and $y$ axes of the right panel indicate mRNA expression analyzed on Affymetrix U133 Plus 2.0 microarrays. (B) Representative positive and negative staining of SIX1 and VEGF-C on human breast cancer tissue sections. Original magnification, $\times 100$. (C) Model depicting the mechanism by which SIX1 promotes metastatic dissemination. Overexpression of SIX1 leads to increased VEGF-C and stimulates lymphangiogenesis, allowing for increased escape of tumor cells through the lymphatics and increased distant metastasis. However, SIX1 is also able to augment the later stages of metastasis of cancer cells that have traveled through the vasculature, thus contributing to metastatic spread via multiple mechanisms. An extension of this finding is that while inhibitors of the VEGF-C/VEGFR3 axis may prevent lymphatic spread, inhibitors of SIX1 are expected to inhibit metastasis at multiple stages, serving as powerful antimetastatic agents.

imaging, whereas the 66cl4-scramble plus pcDNA control group developed significant metastases. Indeed, the 6-thioguanine colony assay did not detect a significant difference in colony formation in cells retrieved from the lungs of mice bearing 66cl4-Six1 KD plus Vegf-c rescue tumors, when compared with mice bearing 66cl4-Six1 KD plus pcDNA tumors (Figure 6B). However, Vegf-c rescue in Six1 KD tumors did lead to an increase in lymphangiogenesis (measure by Lyve1 expression) in the tumors (Figure 6C) as well as an increase in luciferase signal around axillary lymph node regions, in which metastases were confirmed using a 6-thioguanine assay of dissected lymph nodes (Figure 6D and data not shown). Thus, restoration of Vegf-c partially restored lymphangiogenesis and lymph node metastasis in the presence of Six1 KD. Interestingly, in addition to rescuing the lymphatic metastatic phenotype, Vegf-c expression in the context of Six $1 \mathrm{KD}$ also restored luciferase signal of the primary tumors back to that observed in the 66cl4scramble tumors (Supplemental Figure 14), suggesting that Vegf-c is required by the tumor cells for their viability, although it should be noted that it did not fully restore tumor size (data not shown). These data suggest that Vegf-c is important for Six1-induced lym- phangiogenesis and lymphatic metastasis but that it is not sufficient to completely restore lymphangiogenesis to wild-type levels or to restore distant metastasis induced by Six1.

SIX1 and VEGF-C are coexpressed in breast cancer cell lines and in human breast malignancies. Based on our demonstration that SIX1 requires VEGF-C to induce metastasis, we examined whether the correlation between SIX1 and VEGF-C could be extended to additional breast cancer cell lines as well as to human breast cancer. Using Oncomine, expression values for SIX1 and VEGFC were retrieved from the Adai microarray data set (Genentech), and the expression data were plotted to perform a correlation analysis between SIX1 and VEGFC in breast cancer cell lines representing different histologic subtypes of breast cancer (Figure 7A). Spearman analysis performed on the plotted mRNA values for SIX1 and VEGFC demonstrates that there is a significant correlation between the expression of SIX1 and VEGFC in numerous breast cancer cell lines (Spearman $r=0.4162, P=0.0129$ ). It should be noted that expression of SIX1 and VEGFC is also correlated in ovarian cancer cell lines (Supplemental Figure 15), implying that SIX1 may regulate VEGF-C/lymphangiogenesis in these cancers as well. However, 
this observation remains to be experimentally proven. We further performed immunohistochemical analysis for SIX1 and VEGF-C on 110 cases of invasive ductal carcinoma and found a significant correlation between the presence of nuclear SIX1 and cytoplasmic VEGF-C ( $\chi^{2}$ test, $P=0.049$; Figure $7 \mathrm{~B}$ and Supplemental Figure $16)$. Together, these results strongly suggest that the regulation of VEGF-C by SIX 1 is not confined to breast cancer cell lines but does occur in human tumors and provide an explanation for the previous finding that SIX1 expression correlates with lymph node-positive status in human breast cancer (10).

\section{Discussion}

SIX1 is overexpressed in $50 \%$ of primary tumors and in an even greater percentage (90\%) of metastatic lesions, suggesting that it plays a role both in tumor initiation and progression. Indeed, SIX1 is transforming and metastasis-promoting in both transgenic and xenograft mouse models (8-10). SIX1 likely induces transformation via its ability to increase proliferation (6), survival (34), and genomic instability (8). Prometastatic phenotypes induced by SIX1 include augmentation of TGF- $\beta$ signaling, which results in an EMT and is required for SIX1-induced metastasis in an experimental metastasis model, in which late-stage metastasis is assessed. In this study, we demonstrated that in addition to the roles that SIX1 plays in tumorigenesis and in later stages of metastasis, SIX1 also plays an important role in early-stage metastasis, particularly lymphatic metastasis, by inducing VEGF-C and stimulating lymphangiogenesis and lymphatic invasion.

Overexpression of VEGF-C has been implicated in a number of cancers (35-38), and its role in promoting lymphatic metastasis has been demonstrated in several VEGF-C overexpression animal models of mammary carcinoma (25-27). Although growth factors have been found to regulate the expression of VEGF-C $(39,40)$, here, we demonstrate for what we believe to be the first time that SIX1 can directly upregulate VEGFC through transcription and stimulate VEGF-C-mediated lymphangiogenesis in breast cancer cells, thereby contributing to lymphatic dissemination and distant metastasis. In addition, examination of gene expression data sets in Oncomine and staining of breast cancer tissue arrays demonstrates a correlation between SIX1 and VEGF-C in breast cancer.

It should be noted that the ability of SIX1 to regulate VEGF-C does not appear limited to cancers. For example, mouse embryo fibroblasts derived from Six 1 knockout mice had lower Vegf-c levels when compared with wild-type mouse embryo fibroblast cells (Supplemental Figure 17A). Furthermore, if SIX1 is ectopically expressed in normal but immortalized MCF12A mammary epithelial cells, VEGFC levels increased (Supplemental Figure 17B). Together, these data strengthen our hypothesis that SIX1 is a bona fide regulator of VEGF-C. Most importantly, in this study, we provide what we believe to be the first direct mechanistic explanation of how VEGF-C, and thus lymphangiogenesis, is upregulated specifically in breast cancer.

Clinically, solid tumors (such as breast cancer) metastasize in part through the lymphatic system; live imaging of mice with orthotopic breast cancer has demonstrated that tumor cells metastasize to the lymph node (41). Although lymph node metastasis itself is not life threatening, it has an important prognostic value in many types of cancers. However, the mechanism by which tumor cells reach the lymph node is not well understood. While some studies indicate the importance of the interaction among tumor cells and the lymphatic system and the possibility of passive trans- port of tumor cells through preexisting lymphatic vessels (42), growing evidence suggests that tumor-induced lymphangiogenesis is also a mechanism by which tumor cells escape from primary sites. In contrast to tumor-associated angiogenesis, tumor-associated lymphangiogenesis is a relatively new and somewhat controversial field of study. First, the role of newly developed lymphatic vessels in the spread of tumor cells has been questioned due to the fact that tumor-stimulated lymphatic vessels may lack normal function $(43,44)$. In addition, a subset of clinical observations suggest that tumor cells invade into preexisting lymphatic vessels to promote nodal metastases rather than through newly developed lymphatic vessels (45). However, numerous clinical studies demonstrate a correlation between lymphatic vessel density and lymphatic metastasis in breast cancers (15). Moreover, animal studies show that increased lymphangiogenesis correlates with increased lymph node/distant metastasis and, in addition, inhibition of tumor-induced lymphangiogenesis by treatment with the soluble VEGFR-3 receptor protein or VEGFR-3 blocking antibodies suppresses metastasis to RLNs as well as systematic metastases $(25,27$, $46,47)$. In this study, we demonstrate that SIX1-induced lymphangiogenesis is dependent on its ability to induce VEGF-C and that the induction of VEGF-C by SIX1 is critical for its ability to induce lymphatic and distant metastasis. Interestingly, we observe that VEGF-C is necessary, but not sufficient, for SIX1-induced distant metastasis. Indeed, our studies suggest that additional factors regulated by SIX1 may be required to fully promote both lymphangiogenesis as well as to accomplish the later stage of metastasis. Since reconstitution of Vegf-c in $66 \mathrm{cl} 4 \mathrm{KD}$ cells only partially restored lymphangiogenesis, the question of whether lymphangiogenesis is sufficient for distant metastasis is still unanswered. Instead, it is possible that SIX1 influences factors in addition to VEGF-C that enhance lymphangiogenesis and that SIX1 also acts at other points in the metastatic cascade, such as EMT (10). Nonetheless, it is clear from our data that VEGF-C and lymphangiogenesis are critical to the ability of SIX1 to induce metastatic spread.

The presence of tumor cells in the sentinel lymph node is an important indicator for tumor staging and subsequent metastasis. Patients with breast cancer who have lymph node involvement usually require the removal of one or few lymph nodes and may suffer the risk of lymphedema. Our study suggests that antiVEGF-C/VEGFR-3 treatment may particularly benefit patients whose tumors overexpress SIX1, as these patients are likely to have higher rates of lymphangiogenesis. Perhaps more importantly, since SIX1 mediates metastasis by multiple means, including, but not limited to, its ability to promote VEGF-C production and lymphangiogenesis, it may be more attractive to identify means to target SIX1 directly (Figure 7C). Because SIX1 is highly expressed during embryogenesis but relatively silent in normal, differentiated adult mammary tissue, one may expect drugs targeting SIX1 to be potent metastasis suppressors while conferring limited side effects. However, our data suggest that such drugs would likely need to target SIX family members globally, rather than SIX1 specifically, since Six 2 is elevated in metastases derived from tumors in which Six 1 is knocked down. Such redundancy has been observed between Six family members during embryonic development (4, 48-51). Upregulation of Six2 has been observed in the mammary glands of Six 1 knockout mice, suggesting that it may compensate for Six1 loss during postnatal mammary development (52), similar to what we observed in tumors in which Six1 was knocked down. Although the role of SIX2 has not been explored in cancer pro- 
gression, its upregulation has been identified in a bone-metastatic signature using in vivo selection of a metastatic breast cancer cell line (53). Since we did not observe increased Six2 expression in our 66 cl4-Six1 KD cells in vitro, in vivo selection may be critical for compensatory upregulation of Six2. Regardless of the mechanism by which Six2 is expressed in Six1 KD cells, these data suggest a critical role for the SIX family members in metastatic dissemination and pave the way for novel anticancer treatments.

\section{Methods}

Animal studies. In the orthotopic model using MCF7-SIX1 cells, $1 \times 10^{6}$ cells in $100 \mu \mathrm{l}$ of growth factor-reduced Matrigel (BD Biosciences) were injected into the fourth mammary fat pad of 5-week-old female nude mice (Taconic), and tumor growth was measured biweekly using calipers. Prior to cell injection, nude mice were implanted with estrogen pellets, as previously described (10). Mice were sacrificed, and primary tumors were taken for analysis once tumors reached a volume $(V)$ of $2 \mathrm{~cm}^{3}$ - according to the formula $V=1 / 2 \times W^{2} \times L$, where $W$ represents width, and $L$ represents length. Female Balb/c mice ( 6 to 8 weeks of age; obtained from National Cancer Institute) were purchased for our orthotopic metastasis model using $66 \mathrm{cl} 4$ mammary carcinoma cell lines. $1 \times 10^{6}$ cells in $100 \mu \mathrm{l}$ of DMEM medium were injected into the fourth mammary fat pad. Tumor growth was measured biweekly, as outlined above, using calipers, and mice were sacrificed 40 days after injection or when the tumors reached the same size.

Detection of dissemination. Animals injected with Zsgreen-labeled MCF7Ctrl or MCF7-SIX1 cells were dissected under the fluorescence dissecting microscope (Olympus SZX12), which allows us to visualize lymph node metastasis. Mice injected with $66 \mathrm{cl} 4$ cells tagged with luciferase were monitored using the IVIS 200 Imaging System (Caliper Life Sciences). The mice were anesthetized with isoflurane and imaged 10 minutes after D-luciferin injection (Gold Biotechnology). Quantification was performed using LivingImage software version 2.6. Mice were imaged at days 2, 14, 21, 28, and 38 and then sacrificed at day 40 . Lungs from the animals were taken and minced into pieces, treated with collagenase IV and Elastase (Worthington Biochemical), and filtered through $70 \mu \mathrm{m}$ nylon cell strainers (BD Falcon). Cells were resuspended and plated in serial dilution (1:5, 1:10, 1:50, 1:100, $1: 1,000)$ in DMEM growth medium with $60 \mu \mathrm{M}$ 6-thioguanine. After incubating the cells for 10 days in culture, the cells were fixed in $95 \%$ ethanol and stained with crystal violet to perform colony counts.

Experimental procedures. For details on other experimental procedures, see the Supplemental Methods.
Microarray analysis and statistics. Microarray analysis was performed using the Affymetrix GeneChip HT-U133A, as described previously (10). Data were deposited in the NCBI GEO database (accession numbers GSE23655 and GSE35314; http://www.ncbi.nlm.nih.gov/geo). Statistical analysis was performed with 2-tailed $t$ test (Prism 3.0), except when otherwise noted in figure legends. Error bars represent the standard error of the mean. $P$ values of less than 0.05 were considered significant. Statistical analysis was done with the help of Anna Baron, our collaborating biostatistician.

Study approval. Animal protocols performed in this work were approved by the Institutional Animal Care and Use Committee at the University of Colorado Anschutz Medical Campus.

\section{Acknowledgments}

This work was funded by grants from National Cancer Institute (2R01-CA095277 and 1R01-CA157790) and from American Cancer Society (RSG-07-183-01-DDC) to H.L. Ford. C.-A. Wang and D.S. Micalizzi were funded by a predoctoral fellowship from the Department of Defense Breast Cancer Research Program (W81ZWH-101-0162 and W81XWH-06-1-0757). A.N. Patrick was funded by a Pediatric Hematology/Oncology Postdoctoral Fellowship from the NIH (2T32082086-11A1). We thank Fred R. Miller for the 67NR and $66 \mathrm{cl} 4$ cell lines, Kari Alitalo for VEGF-C promoter construct, and Anna Baron for statistical advice. We also thank Jim McManaman for help with microscopy and SlideBook software. Finally, we thank Lina Dimberg for help with ChIP and other members of the Ford laboratory for helpful advice and suggestions.

Received for publication July 11, 2011, and accepted in revised form February 8, 2012.

Address correspondence to: Heide L. Ford, University of Colorado School of Medicine, Anschutz Medical Campus, RC2, Rm. 3100C, Aurora, Colorado 80045, USA. Phone: 303.724.3509; Fax: 303.724.3512; E-mail: Heide.Ford@ucdenver.edu.

Kimberly C. Lemmer's present address is: Great Lakes Bioenergy Research Center, University of Wisconsin-Madison, Madison, Wisconsin, USA.

J. Chuck Harrell's present address is: University of North Carolina at Chapel Hill, Chapel Hill, North Carolina, USA.
1. Seo HC, Curtiss J, Mlodzik M, Fjose A. Six class homeobox genes in drosophila belong to three distinct families and are involved in head development. Mech Dev. 1999;83(1-2):127-139.

2. Li X, et al. Eya protein phosphatase activity regulates Six1-Dach-Eya transcriptional effects in mammalian organogenesis. Nature. 2003;426(6964):247-254.

3. Yu YL, Davicioni E, Triche TJ, Merlino G. The homeoprotein Six 1 transcriptionally activates multiple protumorigenic genes but requires Ezrin to promote metastasis. Cancer Res. 2006;66(4):1982-1989.

4. Kobayashi H, Kawakami K, Asashima M, Nishinakamura R. Six 1 and Six 4 are essential for Gdnf expression in the metanephric mesenchyme and ureteric bud formation, while Six1 deficiency alone causes mesonephric-tubule defects. Mech Dev . 2007;124(4):290-303.

5. Yu Y, Davicioni E, Triche TJ, Merlino G. The homeoprotein six1 transcriptionally activates multiple protumorigenic genes but requires ezrin to promote metastasis. Cancer Res. 2006;66(4):1982-1989.

6. Coletta RD, et al. The Six1 homeoprotein stimulates tumorigenesis by reactivation of cyclin A1.
Proc Natl Acad Sci U S A. 2004;101(17):6478-6483.

7. Micalizzi DS, Wang CA, Farabaugh SM, Schiemann WP, Ford HL. Homeoprotein Six 1 increases TGF-beta type I receptor and converts TGF-beta signaling from suppressive to supportive for tumor growth. Cancer Res. 2010;70(24):10371-10380.

8. Coletta RD, Christensen KL, Micalizzi DS, Jedlicka P, Varella-Garcia M, Ford HL. Six1 overexpression in mammary cells induces genomic instability and is sufficient for malignant transformation. Cancer Res. 2008;68(7):2204-2213.

9. McCoy EL, et al. Six1 expands the mouse mammary epithelial stem/progenitor cell pool and induces mammary tumors that undergo epithelial-mesenchymal transition. J Clin Invest. 2009;119(9):2663-2677.

10. Micalizzi DS, et al. The Six1 homeoprotein induces human mammary carcinoma cells to undergo epithelial-mesenchymal transition and metastasis in mice through increasing TGF-beta signaling. J Clin Invest. 2009;119(9):2678-2690.

11. Yu Y, Khan J, Khanna C, Helman L, Meltzer PS, Merlino G. Expression profiling identifies the cytoskeletal organizer ezrin and the developmental homeoprotein Six-1 as key metastatic regulators. Nat Med. 2004;10(2):175-181.

12. $\mathrm{Ng} \mathrm{KT}$, et al. Suppression of tumorigenesis and metastasis of hepatocellular carcinoma by shRNA interference targeting on homeoprotein Six1. Int J Cancer. 2010;127(4):859-872.

13. Donegan WL. Tumor-related prognostic factors for breast cancer. CA Cancer J Clin. 1997;47(1):28-51.

14. Carter CL, Allen C, Henson DE. Relation of tumor size, lymph node status, and survival in 24,740 breast cancer cases. Cancer. 1989;63(1):181-187.

15. Ran S, Volk L, Hall K, Flister MJ. Lymphangiogenesis and lymphatic metastasis in breast cancer. Pathophysiology. 2010;17(4):229-251.

16. Mandriota SJ, et al. Vascular endothelial growth factor-C-mediated lymphangiogenesis promotes tumour metastasis. EMBO J. 2001;20(4):672-682.

17. Mumprecht V, Detmar M. Lymphangiogenesis and cancer metastasis. J Cell Mol Med. 2009; 13(8A):1405-1416.

18. Bjorndahl $\mathrm{M}$, et al. Insulin-like growth factors 1 and 2 induce lymphangiogenesis in vivo. Proc Natl Acad Sci U S A. 2005;102(43):15593-15598. 
19. Cao R, et al. PDGF-BB induces intratumoral lymphangiogenesis and promotes lymphatic metastasis. Cancer Cell. 2004;6(4):333-345.

20. Mohammed RA, Green A, El-Shikh S, Paish EC, Ellis IO, Martin SG. Prognostic significance of vascular endothelial cell growth factors -A, -C and -D in breast cancer and their relationship with angio- and lymphangiogenesis. BrJ Cancer. 2007;96(7):1092-1100.

21. Nakamura Y, et al. Lymph vessel density correlates with nodal status, VEGF-C expression, and prognosis in breast cancer. Breast Cancer Res Treat. 2005;91(2):125-132.

22. Gu Y, Qi X, Guo S. Lymphangiogenesis induced by VEGF-C and VEGF-D promotes metastasis and a poor outcome in breast carcinoma: a retrospective study of 61 cases. Clin Exp Metastasis. 2008; 25(7):717-725.

23. Karkkainen MJ, et al. Vascular endothelial growth factor $\mathrm{C}$ is required for sprouting of the first lymphatic vessels from embryonic veins. Nat Immunol. 2004;5(1):74-80.

24. Baldwin ME, et al. Vascular endothelial growth factor $\mathrm{D}$ is dispensable for development of the lymphatic system. Mol Cell Biol. 2005;25(6):2441-2449.

25. Karpanen T, et al. Vascular endothelial growth factor $\mathrm{C}$ promotes tumor lymphangiogenesis and intralymphatic tumor growth. Cancer Res. 2001; 61(5):1786-1790.

26. Mattila MM, Ruohola JK, Karpanen T, Jackson DG, Alitalo K, Harkonen PL. VEGF-C induced lymphangiogenesis is associated with lymph node metastasis in orthotopic MCF-7 tumors. Int J Cancer. 2002;98(6):946-951.

27. Skobe $M$, et al. Induction of tumor lymphangiogenesis by VEGF-C promotes breast cancer metastasis. Nat Med. 2001;7(2):192-198.

28. Heanue TA, et al. Synergistic regulation of vertebrate muscle development by Dach2, Eya2, and Six1, homologs of genes required for Drosophila eye formation. Genes Dev. 1999;13(24):3231-3243.

29. Affolter M, Slattery M, Mann RS. A lexicon for homeodomain-DNA recognition. Cell. 2008; 133(7):1133-1135.

30. Maruyama K, et al. Inflammation-induced lymphan- giogenesis in the cornea arises from CD11b-positive macrophages. J Clin Invest. 2005;115(9):2363-2372.

31. Aslakson CJ, Miller FR. Selective events in the metastatic process defined by analysis of the sequential dissemination of subpopulations of a mouse mammary tumor. Cancer Res. 1992;52(6):1399-1405.

32. Cao $\mathrm{Y}$, et al. Vascular endothelial growth factor $C$ induces angiogenesis in vivo. Proc Natl Acad SciUS A. 1998;95(24):14389-14394

33. Miller BE, Roi LD, Howard LM, Miller FR. Quantitative selectivity of contact-mediated intercellular communication in a metastatic mouse mammary tumor line. Cancer Res. 1983;43(9):4102-4107.

34. Behbakht K, et al. Six 1 overexpression in ovarian carcinoma causes resistance to TRAIL-mediated apoptosis and is associated with poor survival. Cancer Res. 2007;67(7):3036-3042.

35. Yang J, et al. Increased expressions of vascular endothelial growth factor (VEGF), VEGF-C and VEGF receptor-3 in prostate cancer tissue are associated with tumor progression. Asian J Androl. 2006; 8(2):169-175

36. Ueda $\mathrm{M}$, et al. Correlation between vascular endothelial growth factor-C expression and invasion phenotype in cervical carcinomas. Int J Cancer. 2002 98(3):335-343.

37. O-charoenrat P, Rhys-Evans P, Eccles SA. Expression of vascular endothelial growth factor family members in head and neck squamous cell carcinoma correlates with lymph node metastasis. Cancer. 2001;92(3):556-568.

38. Kinoshita J, Kitamura K, Kabashima A, Saeki H, Tanaka S, Sugimachi K. Clinical significance of vascular endothelial growth factor-C (VEGF-C) in breast cancer. Breast Cancer Res Treat. 2001;66(2):159-164.

39. Enholm B, et al. Comparison of VEGF, VEGF-B, VEGF-C and Ang-1 mRNA regulation by serum, growth factors, oncoproteins and hypoxia. Oncogene. 1997; 14(20):2475-2483.

40. Tsai PW, Shiah SG, Lin MT, Wu CW, Kuo ML. Upregulation of vascular endothelial growth factor $\mathrm{C}$ in breast cancer cells by heregulin-beta 1 . A critical role of $\mathrm{p} 38 /$ nuclear factor-kappa B signaling pathway. J Biol Chem. 2003;278(8):5750-5759.
41. Dadiani M, et al. Real-time imaging of lymphogenic metastasis in orthotopic human breast cancer. Cancer Res. 2006;66(16):8037-8041.

42. Issa A, Le TX, Shoushtari AN, Shields JD, Swartz MA. Vascular endothelial growth factor-C and $\mathrm{C}-\mathrm{C}$ chemokine receptor 7 in tumor cell-lymphatic cross-talk promote invasive phenotype. Cancer Res. 2009;69(1):349-357.

43. Padera TP, et al. Lymphatic metastasis in the absence of functional intratumor lymphatics. Science. 2002;296(5574):1883-1886.

44. Isaka N, Padera TP, Hagendoorn J, Fukumura D, Jain RK. Peritumor lymphatics induced by vascular endothelial growth factor-C exhibit abnormal function. Cancer Res. 2004;64(13):4400-4404.

45. Williams CS, et al. Absence of lymphangiogenesis and intratumoural lymph vessels in human metastatic breast cancer. J Pathol. 2003;200(2):195-206.

46. He Y, et al. Suppression of tumor lymphangiogenesis and lymph node metastasis by blocking vascular endothelial growth factor receptor 3 signaling. J Natl Cancer Inst. 2002;94(11):819-825.

47. Burton JB, et al. Suppression of prostate cancer nodal and systemic metastasis by blockade of the lymphangiogenic axis. Cancer Res. 2008;68(19):7828-7837.

48. Laclef C, Hamard G, Demignon J, Souil E, Houbron C, Maire P. Altered myogenesis in Six1-deficient mice. Development. 2003;130(10):2239-2252.

49. Laclef C, Souil E, Demignon J, Maire P. Thymus, kidney and craniofacial abnormalities in Six 1 deficient mice. Mech Dev. 2003;120(6):669-679.

50. Ozaki H, et al. Six4, a putative myogenin gene regulator, is not essential for mouse embryonal development. Mol Cell Biol. 2001;21(10):3343-3350.

51. Grifone R, et al. Six1 and Six 4 homeoproteins are required for Pax3 and Mrf expression during myogenesis in the mouse embryo. Development. 2005; 132(9):2235-2249.

52. Coletta RD, et al. Characterization of the Six1 homeobox gene in normal mammary gland morphogenesis. BMC Dev Biol. 2010;10:4

53. Kang Y, et al. A multigenic program mediating breast cancer metastasis to bone. Cancer Cell. 2003; 3(6):537-549. 